\title{
Elastic deformation of a fluid membrane upon colloid binding
}

\author{
Markus Deserno* \\ Department of Chemistry and Biochemistry, UCLA, 405 Hilgard Ave., Los Angeles, California 90095-1569, USA
}

(Received 28 March 2003; published 12 March 2004)

\begin{abstract}
When a colloidal particle adheres to a fluid membrane, it induces elastic deformations in the membrane which oppose its own binding. The structural and energetic aspects of this balance are investigated within the framework of a Helfrich Hamiltonian. Based on the full nonlinear shape equations for the membrane profile, a line of continuous binding transitions and a second line of discontinuous envelopment transitions are found, which meet at an unusual triple point. The regime of low tension is studied analytically using a small gradient expansion, while in the limit of large tension scaling arguments are derived which quantify the asymptotic behavior of phase boundary, degree of wrapping, and energy barrier. The maturation of animal viruses by budding is discussed as a biological example of such colloid-membrane interaction events.
\end{abstract}

DOI: 10.1103/PhysRevE.69.031903

PACS number(s): $87.16 . \mathrm{Dg}, 87.15 . \mathrm{Kg}, 46.70 . \mathrm{Hg}$

\section{INTRODUCTION}

Arguably the most important structural component of all living cells is the phospholipid bilayer. It combines the two diametrical tasks of partitioning-thereby organizing the complex hierarchy of intracellular biochemical environments - while at the same time providing controlled transport mechanisms between neighboring compartments [1]. The size of particles being transported spans several orders of magnitude, ranging all the way from subnanometer ions, whose passage through the bilayer is facilitated by protein channels, up to micron-sized objects engulfed by the large-scale membrane deformations occurring during phagocytosis.

Since cell survival depends on a meticulous balance of these processes, they are actively controlled and require metabolic energy to proceed. Still, there are cases where they happen passively as a result of generic physical interactions, for instance, a sufficiently strong adhesion between the particle about to be transported and the membrane. An example that has been studied in extensive detail is the route along which many animal viruses leave their host cell [2]. After entering the cell (typically via receptor mediated endocytosis or other active processes [3]) and completion of the viral replication steps, the new virions have to get out again. Many virus families accomplish this by the plasma membrane wrapping their nucleoprotein capsid and pinching off ("budding") — a step which not only sets them free but by which they also acquire their final coating. A particularly clean model case is provided by Semliki Forest virus, in which case the binding between capsid and membrane is promoted by viral ("spike") proteins [4-6].

A different realization of such a wrapping event is presumably exploited by an efficient gene transfection system proposed a few years ago by Boussif et al. [7]. There, DNA is complexed by the cationic branched polymer polyethylenimine into a globular complex, which then enters the cell (as deduced from reporter gene expression). Rather than particu-

\footnotetext{
*Present address: Max-Planck-Institut für Polymerforschung, Ackermannweg 10, 55128 Mainz, Germany.
}

lar targeting signals, the slight net positive charge of the complex is believed to trigger adhesion and membrane penetration via an electrostatic interaction with negatively charged regions of the plasma membrane.

Finally, a great deal of biophysical experimental techniques involve the attachment of microbeads to membranes. For instance, one way to measure cellular tensions involves pulling a thin tether with an optical tweezer which grabs a bead adhering to the membrane [8]. Cell membranes and subcellular organelles are routinely probed with an atomic force microscope [9]. And a classical experiment on surface dynamics and locomotion of cells involves monitoring the centripetal motion of a surface adherent particle [10]. In all cases the object adhering to the cell will locally deform its plasma membrane, which can be crucial for interpreting the experimental results. For example, in the case of centripetal bead motion the adhering particles have been observed to actually become engulfed by the cell without involvement of the endocytosis machinery, if only the membrane tension is low enough [11].

Wrapping and budding processes occur very frequently in cells, but even though they are extensively studied experimentally, the high complexity of the real biological situation renders a clear extraction of underlying physical principles very difficult; it is not even obvious or undisputed whether an explanation in terms of such principles is possible. A better look at the physics is therefore provided by more easily controllable experiments on the adsorption of colloids onto model lipid bilayers. The degree of wrapping of a colloid by a giant phospholipid vesicle has, for instance, been studied by Dietrich et al. [12], who showed that it can be quantitatively understood in terms of a balance between adhesion and elastic energy. Colloids will change the shape of the vesicle they adhere to, and this may give rise to attractive interactions between them [13]. If the membrane tension is low enough or the colloid sufficiently small, bending of the membrane will become an important contribution which can be strong enough to completely suppress adhesion [14-16]. The bending stiffness will also prevent any "kink" in the membrane profile at the line of contact; therefore, the notion of a contact angle only remains meaningful in an asymptotic sense and needs to be replaced by the concept of contact 
curvature [17-20]. For vesicles adhering on flat surfaces this can be measured by visualizing the contact zone via reflection interference contrast microscopy [21], but for adhesion of membranes on strongly curved substrates-in particular, small colloids - or if one needs resolution much below optical length scales, this option is not available. Here, computer simulations can provide means for a closeup study [22], but to date it is still difficult to perform a quantitative analysis relating the observed geometry to the underlying mesoscopic elastic properties.

Instead of directly measuring the local deformations, one can study their indirect consequences and from there attempt to deduce some of their properties. This of course requires theoretical modeling for bridging the gap. For instance, lateral membrane tensions can be inferred from the force required to pull a thin tether from the membrane [8]. Recent theoretical work by Derényi et al. shows that the force as a function of tether length is quite subtle and shows an initial oscillatory structure before exponentially approaching the asymptotic value [23]. Very useful information might be obtained from this, for example, an independent estimate of the tether width. For less strongly bound beads Boulbitch has related the unbinding force to the adhesion energy, membrane bending stiffness, and the elasticity of an underlying elastic network (such as the cytoskeleton) by describing the associated membrane deformation within a small gradient approximation [24]. And for the case of colloids adhering to quasispherical vesicles a simple ansatz for the membrane shape has been shown to yield a structural phase diagram indicating when the colloid is free, partially wrapped, and fully enveloped [16].

This paper extends the work of Ref. [25] in developing a detailed theory of the local wrapping behavior in the case of a constant prescribed lateral membrane tension. This "ensemble" is particularly relevant for the biological situation, since almost all cells constantly adjust the amount of lipids in their plasma membrane in order to maintain its lateral tension at some specific set point [26], but it would also apply to a bilayer spread across a hole, provided it is in contact with a lipid reservoir. Tension can even arise from small-scale membrane undulations hiding some membrane area on the larger length scales one might currently be interested in (say, the colloid size) [27]. The situation to be discussed here is similar to the one treated by Boulbitch [24], but it will neither be restricted to small membrane deformations nor to a two-dimensional modeling of the geometry (which would be appropriate for long cylindrical colloids). After setting up the problem and identifying the relevant energies in Sec. II, the full nonlinear shape equations of the membrane profile are studied in Sec. III, leading to the structural wrapping phase diagram. Section IV then treats the small gradient expansion (i.e., the linearized theory), identifies its range of validity as the regime of low membrane tension, and derives various asymptotically exact results. For the opposite regime of large tension several scaling predictions are deduced in Sec. V and validated against the numerical results from Sec. III. In Sec. VI the results obtained throughout the paper are discussed in the framework of the biological example of virus budding.

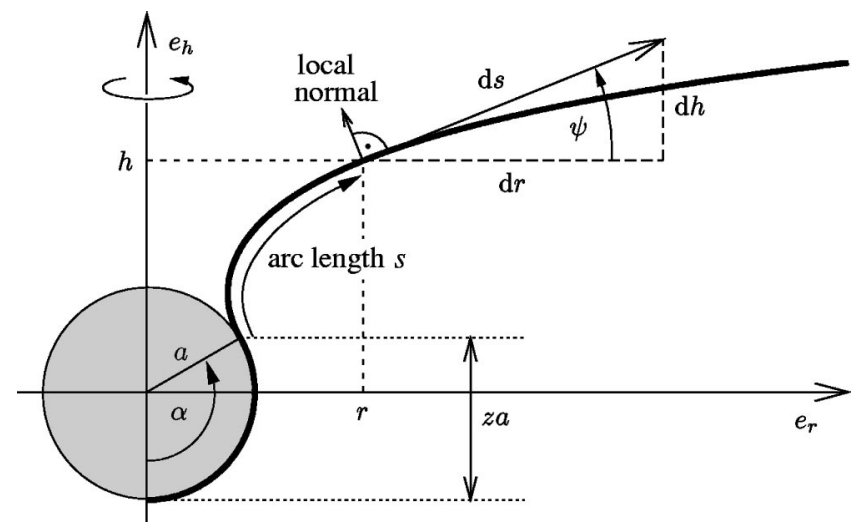

FIG. 1. Illustration of the wrapping geometry and membrane parametrization. An asymptotically flat membrane adheres partially to a spherical colloid of radius $a$ with a degree of wrapping given by $z=1-\cos \alpha$. Cylindrical symmetry around the $e_{h}$ axis is assumed throughout the paper. The possibility of "overhangs" requires going beyond a Monge-like parametrization (which would give the height $h$ as a function of radial distance $r$ ). The choice followed in this work is to specify the angle $\psi$ with respect to the horizontal as a function of arclength $s$ [28].

\section{GENERAL ENERGY CONSIDERATIONS}

The aim of this paper is to describe the local deformations of a flat fluid membrane upon adsorption of a spherical colloid, the geometry of which is depicted schematically in Fig. 1. It will be assumed that this process can be understood as a balance of the following three energy contributions: (i) Adhesion is driven by a contact energy per unit area $w$ and opposed by (ii) the requirement to bend the membrane as well as (iii) the work of pulling excess membrane toward the wrapping site against a prescribed lateral tension $\sigma$. Since the description will not aim at a microscopic understanding, continuum elasticity theory [17] is taken as a basis; in particular, the bending energy per unit area will be described using the standard Helfrich expression [29]

$$
e_{\text {bend }}=\frac{1}{2} \kappa\left(c_{1}+c_{2}-c_{0}\right)^{2}+\bar{\kappa} c_{1} c_{2},
$$

where $c_{1}$ and $c_{2}$ are the local principal curvatures of the two-dimensional membrane surface [30], $c_{0}$ is the spontaneous curvature of the membrane, and $\kappa$ and $\bar{\kappa}$ are elastic moduli (with units of energy). In the following a symmetric membrane is assumed, i.e., $c_{0}=0$ (consistent with the assumption of asymptotic flatness); and since no topological changes will be considered, the second term in Eq. (1) can be dropped [31]. The tension work is per definition given by the lateral tension $\sigma$ times the excess area pulled toward the wrapping site [32]. Note that from tension and bending modulus one can construct a length $\lambda$-specific to the membrane-according to

$$
\lambda:=\sqrt{\frac{\kappa}{\sigma}} .
$$

Membrane deformations on a length scale smaller than $\lambda$ predominantly cost bending energy, while deformations on a 
larger scale pay mostly in tension. An interesting situation occurs when the two intrinsic lengths of the problem - the colloid radius $a$ and the membrane length $\lambda$-are of the same order, because then bending and tension contributions are comparable. In fact, for a typical cellular membrane tension of $\sigma \simeq 0.02 \mathrm{dyn} / \mathrm{cm}[26]$ and a typical bilayer bending modulus of $\kappa \simeq 20 k_{\mathrm{B}} T$ (where $k_{\mathrm{B}} T$ is the thermal energy) one obtains $\lambda \simeq 64 \mathrm{~nm}$ [33]. Viral capsids are about this big [2], therefore this biological situation sits squarely in the crossover regime.

Assuming that the adhering membrane remains in the fluid state, its energy can be calculated easily once the degree of wrapping, $z=1-\cos \alpha$, is specified, because the shape of the membrane is known (see Fig. 1 for the definition of all geometric variables). The area of the colloid covered by membrane is given by $A_{\mathrm{ad}}=2 \pi a^{2} z$, which gives an adhesion energy of $E_{\mathrm{ad}}=-w A_{\mathrm{ad}}=-2 \pi a^{2} z w$. Using Eq. (1), the bending energy is found to be $E_{\text {bend }}=\frac{1}{2} \kappa(1 / a+1 / a)^{2} A_{\text {ad }}$ $=4 \pi z \kappa$. Finally, the work done against a lateral tension $\sigma$ is proportional through $\sigma$ to the excess area pulled toward the wrapping site, which is $\Delta A_{\mathrm{ad}}=\pi a^{2} z^{2}$, giving the tension energy $E_{\text {ten }}=\pi a^{2} z^{2} \sigma$.

It turns out to be advantageous to measure energies in units of the bending constant $\kappa$ and lengths in units of the colloid radius $a$. This suggests the definition of the following three dimensionless variables:

$$
\widetilde{E}:=\frac{E}{\pi \kappa}, \quad \widetilde{w}:=\frac{2 w a^{2}}{\kappa}, \quad \widetilde{\sigma}:=\frac{\sigma a^{2}}{\kappa}=\left(\frac{a}{\lambda}\right)^{2},
$$

where numerical factors of $\pi$ and 2 have been introduced for later convenience. Note that the crossover relation $\lambda \simeq a$ corresponds to $\widetilde{\sigma} \simeq 1$. In terms of these reduced variables the total energy of the colloid-membrane complex is given by

$$
\widetilde{E}=-(\widetilde{w}-4) z+\widetilde{\sigma} z^{2}+\widetilde{E}_{\text {free }}(z, \widetilde{\sigma}),
$$

where $\widetilde{E}_{\text {free }}=E_{\text {free }} / \pi \kappa$ is the (dimensionless) energy of the free part of the membrane. This term is not so easy to calculate, since the membrane shape is not known a priori. Even though the main purpose of this paper is to determine $E_{\text {free }}$ and understand its implications on the wrapping process, it still proves instructive to have a brief look at the problem while ignoring $E_{\text {free }}$. Minimizing Eq. (4) with respect to $z$ (and not forgetting possible boundary minima at $z=0$ or $z$ $=2$ ), the following picture emerges: Colloids do not adhere if $\widetilde{w}<4$, since they cannot pay the bending price. Once $\widetilde{w}$ $>4$, they start to adhere by first being partially wrapped. Full envelopment occurs only if $\widetilde{w}>4+4 \widetilde{\sigma}$. In between, the degree of partial wrapping is $z=(\widetilde{w}-4) / 2 \widetilde{\sigma}$, and both transitions from free to partially wrapped as well as from partially wrapped to fully enveloped are continuous.

There is one case in which the simplification $E_{\text {free }}=0$ in fact holds rigorously, and that is the case of zero tension $\sigma=0[14,15]$. This can be seen as follows: Consider the twoparameter family of cylindrically symmetric surfaces $r(h) / a=a_{1} \cosh \left[\left(h / a-a_{2}\right) / a_{1}\right]$, which are called catenoids. The particular choice $a_{1}=z(2-z)$ and $a_{2}=z-1+\frac{1}{2} z(2$ $-z) \ln [z /(2-z)]$ is readily seen to describe a surface which smoothly touches the colloid with a degree of wrapping equal to $z$. The important point is that such catenoids are minimal surfaces which have zero mean curvature at every point [30], and hence no bending energy. Since (i) for $\sigma=0$ the only possible energy of the free membrane comes from bending and (ii) the bending energy is positive definite, the catenoid is in fact the minimum energy shape. The above simplified description of the problem is thus exact in the case $\sigma=0$, i.e., for $\widetilde{w}<4$ the colloid is not wrapped and for $\widetilde{w}$ $>4$ it is completely wrapped, with no energy barrier impeding the transition [34].

\section{THE FULL SOLUTION OF THE SHAPE PROFILE}

By setting $E_{\text {free }}=0$ one neglects, in particular, any potential effect that the strongly curved part of the membrane close to the line of contact may have on the wrapping behavior. As a next step it is therefore tempting to approximate $\widetilde{E}_{\text {free }}$ by a phenomenological line energy [34]. However, neither the relation between the line tension constant and the membrane properties $\kappa$ and $\sigma$ would be known nor is the implied dependency on the degree of wrapping - namely $\sim \sin \alpha=\sqrt{z(2-z)}$ - supported by more careful studies. (On a scaling level and in the high tension limit this question is revisited in Sec. V.)

In order to draw sound conclusions, an explicit treatment of the membrane deformation is needed. Three approaches are discussed in the following: In the present section the equilibrium membrane shape is determined by minimizing its energy. Since this leads to complicated nonlinear differential equations, one largely has to rely on numerical solutions here. In the second approach, discussed in Sec. IV, a restriction to small membrane deformations renders these differential equations linear, and they can be solved exactly then. However, the latter approach per construction is limited to fairly small degrees of wrapping. Hence, its range of validity is not obvious and has to be checked against the nonlinear results. Finally, in Sec. V a scaling analysis is used to shed light onto the high tension regime.

\section{A. Energy functional and shape equations}

The energy of the free membrane is the surface integral over the local bending and tension contributions and is thus a functional of the shape. Using the cylindrically symmetric angle-arclength parametrization from Fig. 1, the two principal curvatures are found to be $(\sin \psi) / r$ and $\dot{\psi}$, where the dot indicates a derivative with respect to the arclength $s$. The energy functional can then be written as [35-37]

$$
\widetilde{E}_{\text {free }}=\int_{0}^{\infty} d s L\left(\psi, \dot{\psi}, r, \dot{r}, \dot{h}, \lambda_{r}, \lambda_{h}\right),
$$

where the Lagrange function $L$ is defined by

$$
\begin{aligned}
L= & r\left\{\left(\dot{\psi}+\frac{\sin \psi}{r}\right)^{2}+\frac{2 \widetilde{\sigma}}{a^{2}}(1-\cos \psi)\right\} \\
& +\lambda_{r}(\dot{r}-\cos \psi)+\lambda_{h}(\dot{h}-\sin \psi) .
\end{aligned}
$$


The expression in curly brackets contains the bending and tension contributions, while the two additional terms enforce the nonholonomic parametrization constraints $\dot{r}=\cos \psi$ and $\dot{h}=\sin \psi$ by means of the Lagrange parameter functions $\lambda_{r}(s)$ and $\lambda_{h}(s)$. Measuring all lengths in units of $a$, it can be verified that $\widetilde{E}_{\text {free }}$ depends parametrically on $\widetilde{\sigma}$ (and through the boundary conditions on z) but on nothing else. Therefore, $\widetilde{w}$ and $\widetilde{\sigma}$ remain the relevant axes for a structural phase diagram, and no new independent variable is introduced by the free part of the membrane. For the same reason the membrane shape scales with the colloid size $a$ at given $\widetilde{\sigma}$ and $z$.

The Lagrangian $L$ is independent of the arclength $s$, therefore the corresponding Hamiltonian is conserved. Moreover, numerically one usually integrates systems of first-order differential equations. Both observations suggest to switch to a Hamiltonian description. After defining the conjugate momenta

$$
\begin{gathered}
p_{\psi}=\frac{\partial L}{\partial \dot{\psi}}=2 r\left(\dot{\psi}+\frac{\sin \psi}{r}\right), \\
p_{r}=\frac{\partial L}{\partial \dot{r}}=\lambda_{r},
\end{gathered}
$$

and

$$
p_{h}=\frac{\partial L}{\partial \dot{h}}=\lambda_{h},
$$

a Legendre transform yields

$$
\begin{aligned}
H & =\dot{\psi} p_{\psi}+\dot{r} p_{r}+\dot{h} p_{h}-L \\
& =\frac{p_{\psi}^{2}}{4 r}-\frac{p_{\psi} \sin \psi}{r}-\frac{2 \widetilde{\sigma} r}{a^{2}}(1-\cos \psi)+p_{r} \cos \psi+p_{h} \sin \psi .
\end{aligned}
$$

The shape equations, which express the minimization of the functional (5), are the associated Hamilton equations:

$$
\begin{gathered}
\dot{\psi}=\frac{p_{\psi}}{2 r}-\frac{\sin \psi}{r}, \\
\dot{r}=\cos \psi, \\
\dot{h}=\sin \psi, \\
\dot{p}_{\psi}=\left(\frac{p_{\psi}}{r}-p_{h}\right) \cos \psi+\left(\frac{2 \widetilde{\sigma} r}{a^{2}}+p_{r}\right) \sin \psi, \\
\dot{p}_{r}=\frac{p_{\psi}}{r}\left(\frac{p_{\psi}}{4 r}-\frac{\sin \psi}{r}\right)+\frac{2 \widetilde{\sigma}}{a^{2}}(1-\cos \psi),
\end{gathered}
$$

and

$$
\dot{p}_{h}=0 .
$$

Shape equations of this kind have been studied extensively in the past, leading, among many other things, to a very detailed understanding of vesicle conformations (in which case one also needs to fix surface and volume by additional Lagrange multipliers). For a detailed review on this subject see Ref. [37].

\section{B. Boundary conditions}

The situation to be studied is a colloid wrapped by an initially flat membrane. The boundary conditions thus have to ensure that the membrane touches the colloid smoothly and becomes asymptotically flat at large radial distances. At contact, $s=0$, the following must evidently hold (see again Fig. 1):

$$
\begin{gathered}
r(0)=a \sin \alpha, \\
h(0)=-a \cos \alpha,
\end{gathered}
$$

and

$$
\psi(0)=\alpha .
$$

The notion of asymptotic flatness can be enforced by requiring the angle $\psi(s)$ and all of its derivatives to vanish in the limit $s \rightarrow \infty$ [38]. However, it suffices to demand this for the angle $\psi$ and the meridinal curvature $\dot{\psi}$,

$$
\lim _{s \rightarrow \infty} \psi(s)=0
$$

and

$$
\lim _{s \rightarrow \infty} \dot{\psi}(s)=0,
$$

which ensures that tension and curvature energy density vanish if one moves away from the site where the membrane shape is perturbed by the adhering colloid. If $\psi(s)$ vanishes sufficiently rapidly (as it does for $\sigma>0$, see Sec. IV), all contributions beyond some large distance $S$ in arclength will be negligible. A convenient way to exploit this is the following: Choose an upper arclength $S$ and impose the zero angle condition there. Hence, variations of $S$ and $\psi(S)$ are not permitted during functional minimization, but $r(S)$ and $h(S)$ are still free. This implies the additional boundary conditions $[36,39]$

$$
0=\left.\frac{\partial L}{\partial \dot{r}}\right|_{s=S}=p_{r}(S)
$$

and

$$
0=\left.\frac{\partial L}{\partial \dot{h}}\right|_{s=S}=p_{h}(S) .
$$

The Hamilton equation (9f) shows $p_{h}$ to be an integral of "motion," and the boundary condition (12b) fixes its value to zero. Hence, $p_{h}$ drops out of the problem everywhere. The 
condition on $p_{r}$ can be made very useful by a little more thought. Since for $\widetilde{\sigma}>0$ the angle $\psi$ converges to zero in an essentially exponential way (see Sec. IV), the expression for the Hamiltonian will converge toward $p_{r}$. Thus, the requirement of a flat profile implies $H=H(S) \rightarrow p_{r}(S)=0$, or in other words, if the membrane is to become flat, the Hamiltonian must be zero. Using Eqs. (7a) and (8), this can be turned into a condition for $p_{r}$ at the contact boundary:

$$
a p_{r}(0)=\frac{\sqrt{z(2-z)}}{1-z}\left\{1+2 \widetilde{\sigma} z-[a \dot{\psi}(0)]^{2}\right\}
$$

The only remaining variable for which the contact value is not yet known is $p_{\psi}$, or alternatively $\dot{\psi}$. At this point a bit of care is required. As is known, the balance between adhesion energy and elastic membrane deformation results in a boundary condition on the contact curvature [17]. For spherical surfaces this becomes [18]

$$
a \dot{\psi}_{0}:=a \dot{\psi}(0)=1-\sqrt{\widetilde{w}}
$$

However, it is crucial to understand that this condition only holds for the final equilibrium shape of the complex. In the present case the situation is different, because the aim is to calculate the energy $E_{\text {free }}$ at any given value of the penetration $z$. Therefore the adhesion balance is constrained, and Eq. (14) generally will not hold. Nevertheless, by later imposing $\partial E / \partial z=0$ for identifying the equilibrium degree of penetration, Eq. (14) is recovered. This is clarified in the Appendix.

Rather than Eq. (14), it is the condition of asymptotic flatness that will determine $\dot{\psi}_{0}$. In practice, this can be done via a shooting method: For a trial value of $\dot{\psi}_{0}$ integrate the profile and find the arclength $s_{0}$ at which the angle first vanishes, i.e., $\psi\left(s_{0}\right)=0$. Now adjust $\dot{\psi}_{0}$ iteratively and search for the value(s) at which $s_{0}$ diverges. One thereby finds the contact curvature as a function of penetration, $\dot{\psi}_{0}(z)$, in what amounts to a nonlinear eigenvalue problem.

At this point a side note seems appropriate. It turns out that at $\widetilde{\sigma}=\widetilde{\sigma}_{\mathrm{c}}=4.721139 \ldots$ the nature of this eigenvalue problem changes qualitatively, since a region of $z$ values emerges (at $z_{\mathrm{c}}=1.86289 \ldots$ ) for which there exist three contact curvatures which yield profiles satisfying all boundary conditions [the function $\dot{\psi}_{0}(z)$ develops an S shape]. The correct solution has to be identified based on the criterion of lowest energy. For somewhat larger values of $\widetilde{\sigma}$ the lowest energy solution cannot even be realized physically: The corresponding curvature $\dot{\psi}_{0}$ may become larger than $1 / a$, which is geometrically impossible because the membrane cannot bend into the colloid it adheres to. This, however, is not a problem in the present case of an adhesion balance, since the curvature boundary condition from Eq. (14) anyway requires the equilibrium contact curvature to be smaller than $1 / a$. Therefore, the accessible range of multivalued contact curvatures always turns out to lie in between the transition from partially to fully wrapped, and it has no direct consequences on the phase diagram [40]. Still, the mathematical properties

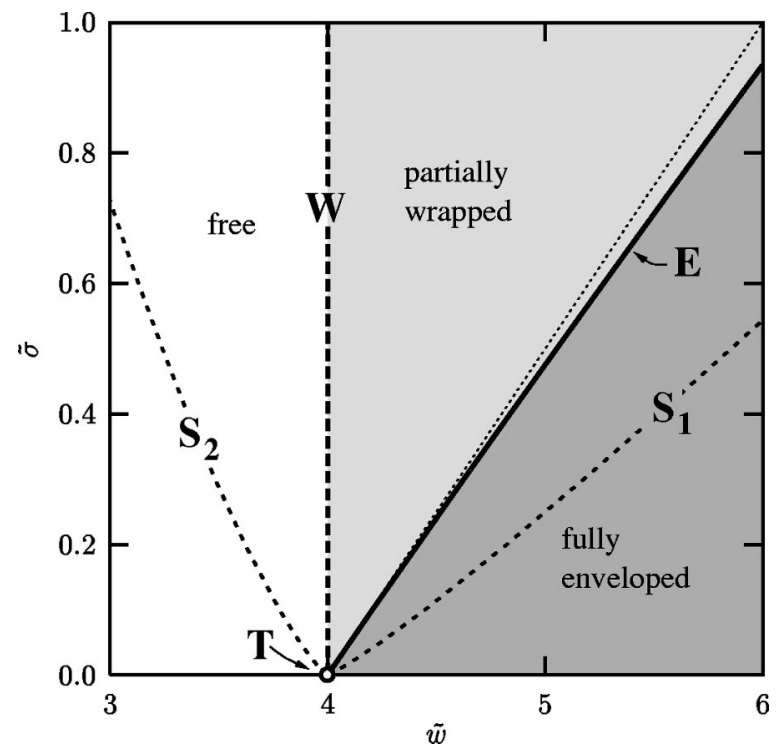

FIG. 2. Structural wrapping phase diagram in the plane of reduced adhesion constant $\widetilde{w}$ and reduced lateral tension $\widetilde{\sigma}$, in the low tension regime $\widetilde{\sigma}<1$ close to the triple point " $\mathrm{T}$ " $(\widetilde{w}=4, \widetilde{\sigma}=0)$. The dashed line "W" marks the continuous transition at which partial wrapping sets in, the bold solid line " $E$ " indicates the discontinuous transition between partially wrapped and fully enveloped, and the short dashed lines " $\mathrm{S}_{1}$ " and " $\mathrm{S}_{2}$ " are the spinodals belonging to "E." The fine dotted line $\widetilde{w}=4+2 \widetilde{\sigma}$ close to E indicates where the fully wrapped state has zero energy.

of this bifurcation may permit some insight into the general nature of the solution, which, however, will not be pursued in the present paper.

\section{Structural phase diagram}

Numerically performing the calculations indicated above yields the shape profile and hence, via Eq. (5), the free membrane energy for any given value of $\widetilde{\sigma}$ and $z$. From Eq. (4) one then determines the total energy as a function of $\widetilde{\sigma}, \widetilde{w}$, and $z$. The minimum in $z$ within the range $[0 ; 2]$ corresponds to the equilibrium state and one obtains the phase diagram as depicted in Fig. 2 [25].

The transition from free to partially wrapped (see dashed line "W") remains unchanged compared to the case where $E_{\text {free }}$ was neglected, because-just like the tension term-the energy of the free membrane is of higher than linear order for small $z$ (this again follows analytically from a small gradient expansion, see Sec. IV). The physics is thus determined by a balance between bending and adhesion alone. However, the transition from partial wrapping to full envelopment (the solid line "E") changes significantly: As can be seen in Fig. 3 , an energy barrier separates the fully and partially wrapped states, rendering the transition discontinuous. This energy barrier turns out to be mostly tension (not bending) energy stored in the free membrane of partially wrapped colloids, and its height can be quite substantial. For instance, with $\sigma=0.02 \mathrm{dyn} / \mathrm{cm}$ (a typical value for a cellular tension [26]), $a=30 \mathrm{~nm}$ (capsid radius of Semliki Forest virus, as an example for a colloidal particle to be wrapped) and $\kappa$ 


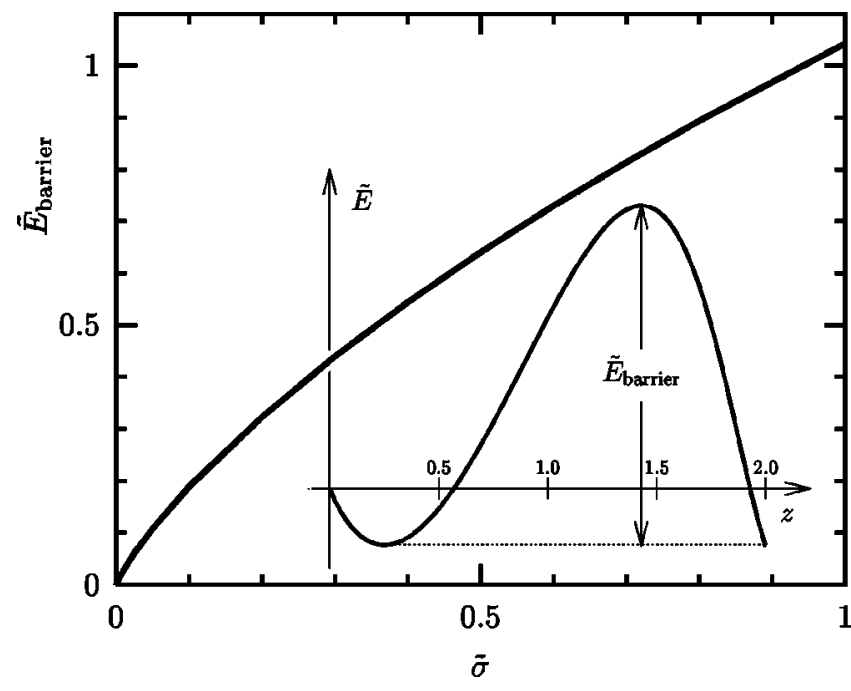

FIG. 3. Height of the energy barrier $\widetilde{E}_{\text {barrier }}=E_{\text {barrier }} / \pi \kappa$ as a function of reduced tension in the low tension regime $\widetilde{\sigma}<1$, at the value of $\widetilde{w}$ where the transition from partial wrapping to full envelopment occurs. The inset illustrates the shape of the function $\widetilde{E}(z)$ and defines the concomitant energy barrier $(\widetilde{\sigma}=0.5$ in this example).

$=20 k_{\mathrm{B}} T$, one finds $\widetilde{\sigma} \approx 0.22$ and from that $E_{\text {barrier }} \approx 22 k_{\mathrm{B}} T$. This barrier is too large to be overcome by thermal fluctuations alone [41]. However, upon increasing the adhesion energy $\widetilde{w}$ and thereby going deeper into the region of full envelopment, the energy barrier separating the partially and the fully wrapped state decreases, ultimately vanishing at the spinodal line " $\mathrm{S}_{1}$ " [42]. Conversely, once the colloid is fully wrapped, the same energy barrier prevents the unwrapping transition, and one has to decrease the value of $\widetilde{w}$ further in order to remove this barrier-see the second spinodal " $\mathrm{S}_{2}$ " in Fig. 2. Cycling across the envelopment transition E thus gives rise to hysteresis, as is illustrated for the particular case $\widetilde{\sigma}=1$ in Fig. 4, for which the energy barrier is $E_{\text {barrier }}$ $\approx 66 k_{\mathrm{B}} T$ using the same system properties as above. Interestingly, this pronounced hysteresis entirely skips the partially wrapped region upon unbinding.

Both $\widetilde{w}$ and $\widetilde{\sigma}$ are proportional to $a^{2}$; therefore, a scan of the particle radius $a$ at fixed values of $\kappa, \sigma$, and $w$ yields lines in the phase diagram which pass through the origin. A careful study of the envelopment boundary $\mathrm{E}$ then reveals that for $w / \sigma \lesssim 1.37$ particles will not become fully enveloped, irrespective of their size, while for $w / \sigma \geqslant 2$ all sufficiently large particles are enveloped. In the small region in between, $1.37 \leqslant w / \sigma \leqslant 2$, particles are only enveloped if they are neither too small nor too large. The asymptotic envelopment condition for small $a$ coincides with the boundary at which wrapping sets in, which is $a=\sqrt{2 \kappa / w}$ or $w / \sigma=2(\lambda / a)^{2}$ $[14,15]$. At the onset of the possibility of full envelopment, $w / \sigma \simeq 1.37$, the first particles to be enveloped have a radius $a \simeq 4.4 \lambda$. Figure 5 summarizes these results.

The energy of the free membrane, $E_{\text {free }}$, vanishes not only in the limit $z \rightarrow 0$ but also in the full-wrapping limit $z \rightarrow 2$. This is reminiscent of the case of an ideal neck connecting two vesicles [43] and relies on the membrane shape locally

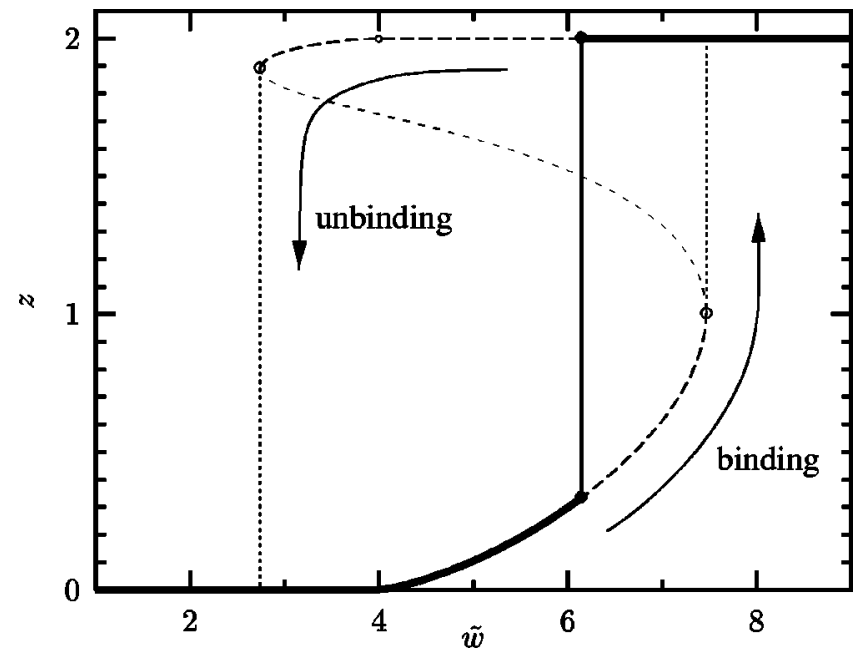

FIG. 4. Hysteresis loop of crossing the envelopment transition by changing the adhesion energy $\widetilde{w}$ for the particular tension $\widetilde{\sigma}$ $=1$. At $\widetilde{w}=4$ binding sets in, at $\widetilde{w} \approx 6.1$ the fully enveloped state becomes stable. However, only at $\widetilde{w} \approx 7.5$ does the huge energy barrier of $66 k_{\mathrm{B}} T$ separating it from the partially wrapped state vanish. On the unbinding branch the system again remains metastable beyond the actual transition, the stable partially wrapped branch of low bindings is entirely skipped and "replaced" by a metastable partially wrapped branch below $\widetilde{w}=4$, featuring large values of $z$. At $\tilde{w} \approx 2.7$ the energy barrier for unbinding vanishes. The unstable branch is indicated by the fine dashed back-bending curve and corresponds to the maximum in $\widetilde{E}(z)$.

approaching a catenoid [44]. This fact is very convenient because knowledge of the exact energy of the enveloped state greatly simplifies the discussion of the structural transitions. This will be further exploited in Sec. IV. As an immediate consequence, it becomes possible to estimate the point of envelopment by comparing the known energy of the fully enveloped state not with the partially wrapped state but simply with the free state, namely, $E=0$. This gives the boundary $\widetilde{w}=4+2 \widetilde{\sigma}$, which is also plotted in Fig. 2 and

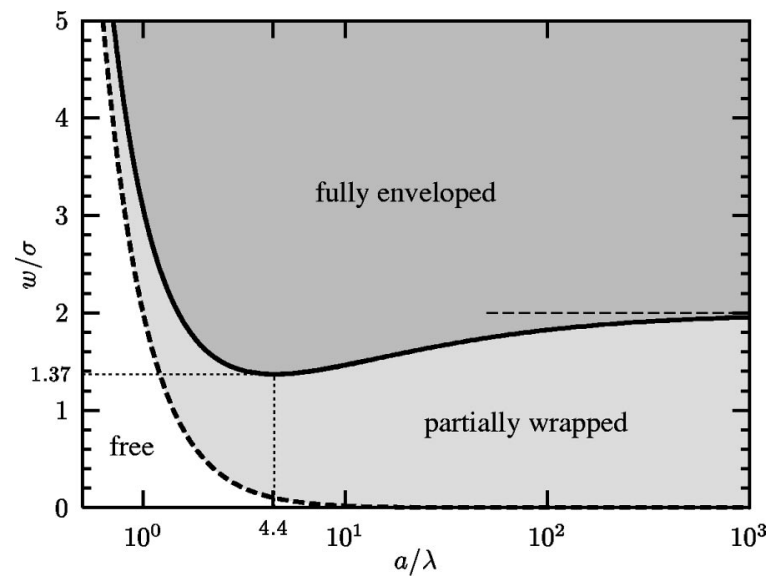

FIG. 5. Influence of the particle radius $a$ on the wrapping behavior. Sufficiently large particles will always at least partially wrap. In the range $1.37 \leqslant w / \sigma \leqslant 2$ particles only become enveloped if they are neither too small nor too large. 


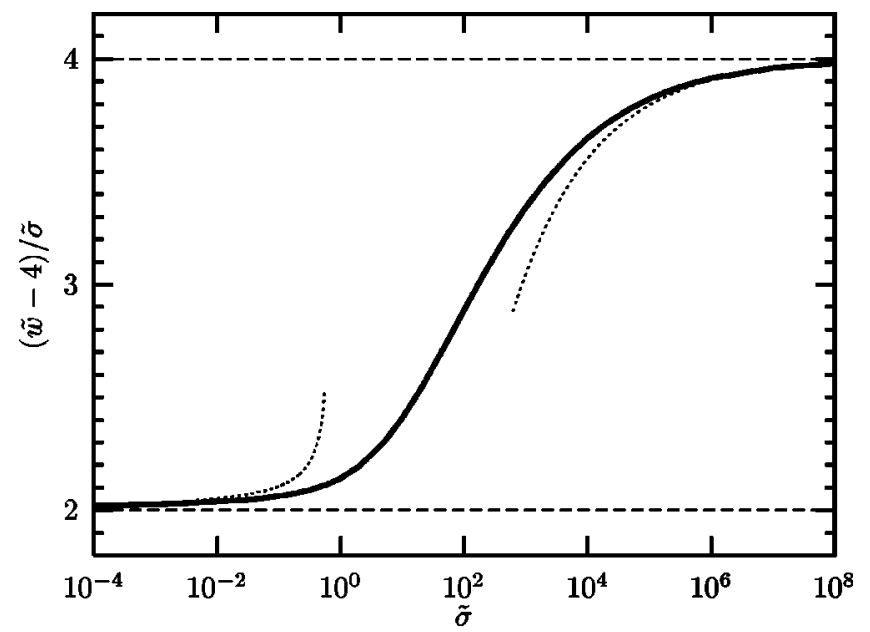

FIG. 6. Phase boundary between partially and fully wrapped state plotted against decades of reduced tension $\widetilde{\sigma}$. The combination $(\widetilde{w}-4) / \widetilde{\sigma}$ is seen to crossover from the value 2 at small $\widetilde{\sigma}$, which follows from the small gradient expansion, to the Young-Duprélimit 4, which results when the energy $E_{\text {free }}$ of the free membrane is negligible. The two dotted curves at small and large $\widetilde{\sigma}$ correspond to the small gradient estimate (27) and the scaling prediction (35), respectively.

which actually becomes asymptotic to the real phase boundary in the limit $\widetilde{\sigma} \rightarrow 0$ (in a complicated logarithmic fashion). Note that this line differs from the phase boundary of the case where $E_{\text {free }}$ had been neglected by a factor 2 in the slope (i.e., the prefactor of $\widetilde{\sigma}$ ) - and in a maybe unexpected way: The region in the phase diagram belonging to fully enveloped states grows at the expense of partially wrapped states. Even though bending and tension energy work against adhesion, they can actually promote envelopment, because partially wrapped states with a large penetration can lower their energy by completing the wrapping, which provides another means to understand why the transition is discontinuous [45]. The same has been found for colloids adhering to quasispherical vesicles [16].

For increasing $\widetilde{\sigma}$ the bending energy should ultimately become negligible compared to the tension. Indeed, in the limit $\kappa \rightarrow 0$ the term $E_{\text {free }}$ vanishes, because the membrane is flat immediately after detaching (smoothness of the slope is no longer required). The equilibrium penetration of partially wrapped colloids, as deduced from Eq. (4), is then $z$ $=\tilde{w} / 2 \widetilde{\sigma}$. This equation can be rewritten as $w=\sigma[1+\cos (\pi$ $-\alpha)]$ and is thereby recognized as the Young-Dupré equation [46], which relates adhesion and tension to the contact angle, here $\pi-\alpha$. The envelopment boundary E consequently occurs at $\widetilde{w}=4 \widetilde{\sigma}$, i.e., where the penetration is $z=2$, or, equivalently, where the contact angle vanishes and the membrane completely "wets" the colloid.

On the basis of these results one expects a crossover from the small gradient asymptotic phase boundary $\widetilde{w}=4+2 \widetilde{\sigma}$, valid below $\widetilde{\sigma} \simeq 1$, to a large tension limit $\widetilde{w}=4 \widetilde{\sigma} \simeq 4+4 \widetilde{\sigma}$. Figure 6 confirms this. However, it is quite remarkable how many orders of magnitude of variation of the reduced tension it takes to establish the transition toward the high tension asymptotic: At $\widetilde{\sigma}=1$ the curve is about 0.142 away from the zero tension asymptotic; getting as close as that to the high tension asymptotics requires $\widetilde{\sigma} \simeq 2 \times 10^{5}$. Over the intervening five orders of magnitude of reduced tension, the influence of bending and tension cannot be easily disentangled. In Sec. V it will be shown that the Young-Dupre limit is reached in a power law fashion with a small exponent $1 / 3$, which partly explains this slow crossover.

It may be worth pointing out that the large range of values of $\widetilde{\sigma}$ is not experimentally unreasonable, because each of the three variables entering the reduced tension can vary by a few orders of magnitude: Membrane tensions between 0.01 $\mathrm{dyn} / \mathrm{cm}$ and $1 \mathrm{dyn} / \mathrm{cm}$ are typical [26], as are bending constants between $1 k_{\mathrm{B}} T$ and $100 k_{\mathrm{B}} T$ [47]. Assuming colloidal radii between $20 \mathrm{~nm}$ and $2 \mu \mathrm{m}$ yields a range for $\widetilde{\sigma}$ from about $10^{-2}$ up to $10^{6}$.

\section{SMALL GRADIENT EXPANSION}

One particular result from the numerical solution of the nonlinear problem is the following: For sufficiently small tension the equilibrium penetration shortly before envelopment ensues is quite small (see the inset in Fig. 3), as is the concomitant perturbation of the flat membrane. Therefore, this region of the phase diagram should be amenable to an approximate treatment of the differential equations which corresponds to a lowest order expansion around the flat profile.

\section{A. Functional and linear shape equations}

If the shape of the membrane is only weakly perturbed, a Monge representation giving the profile height $h$ as a function of the position $\boldsymbol{r}=(x, y)$ in the reference plane is applicable. Bending plus tension energy can then be written as

$$
E=\int d^{2} r \sqrt{1+(\boldsymbol{\nabla} h)^{2}}\left\{\frac{\kappa}{2}\left[\boldsymbol{\nabla} \cdot \frac{\boldsymbol{\nabla} h}{\sqrt{1+(\nabla h)^{2}}}\right]^{2}+\sigma\right\},
$$

where $\boldsymbol{\nabla}$ is the two-dimensional nabla operator in the reference plane. Expanding the two terms in the integrand up to lowest order in $\boldsymbol{\nabla} h$ gives the small gradient expansion of the energy functional

$$
E=\int d^{2} r\left\{\frac{\kappa}{2}\left(\nabla^{2} h\right)^{2}+\frac{\sigma}{2}(\nabla h)^{2}\right\} .
$$

The functional variation $\delta E=0$ finally yields the linear shape equation [48]:

$$
\nabla^{2}\left(\nabla^{2}-\lambda^{-2}\right) h=0
$$

where $\lambda$ is the length introduced in Eq. (2).

\section{B. Equilibrium profile and energy}

The differential equation (17) is solved by eigenfunctions of the Laplacian corresponding to the eigenvalues 0 and $\lambda^{-2}$. In the present cylindrical symmetry the general solution can therefore be written as 


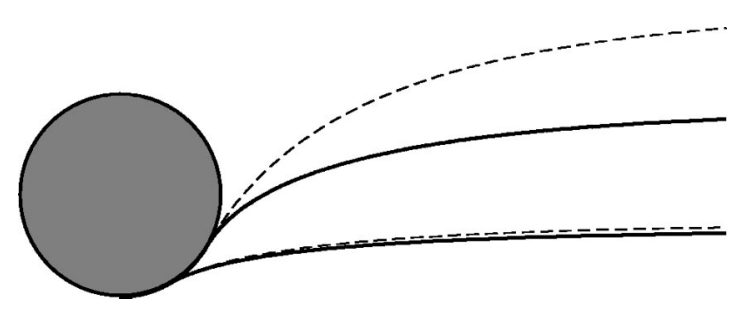

FIG. 7. Exact membrane profiles (solid curves) and small gradient approximation (dashed curves) for two fixed detachment angles $\alpha=30^{\circ}$ and $\alpha=60^{\circ}$. The reduced tension is $\widetilde{\sigma}=0.1$.

$$
h(r)=h_{1}+h_{2} \ln (r / \lambda)+h_{3} K_{0}(r / \lambda)+h_{4} I_{0}(r / \lambda)
$$

where $K_{0}$ and $I_{0}$ are modified Bessel functions [49]. Since $I_{0}(r)$ diverges as $r \rightarrow \infty$, the condition of a flat profile requires $h_{4}=0$. And the coefficient $h_{2}$ has to vanish, because otherwise the energy density is not integrable for $r \rightarrow \infty$ (and $\widetilde{\sigma}>0$ ). The two remaining coefficients are obtained by fixing height and slope of the profile at the point where it touches the colloid. A straightforward calculation then gives the small gradient profile

$$
\frac{h(r)}{a}=z-1+\frac{\lambda}{a} \frac{k}{1-z} \frac{K_{0}(k a / \lambda)-K_{0}(r / \lambda)}{K_{1}(k a / \lambda)},
$$

where the abbreviation $k=\sin \alpha=\sqrt{z(2-z)}$ has been used. Figure 7 illustrates how the small gradient prediction of the profile compares to the full solution. If the detachment angle $\alpha$ is sufficiently small, the overall membrane deformation remains also small, and the profile from the linearized theory follows the full solution quite accurately. However, for a somewhat larger $\alpha$ significant deviations appear: the membrane deformation is predicted to be substantially larger than it actually is. It is worth pointing out that a good understanding of the profile is important if one attempts to infer physical properties of the membrane or the complex by measuring the membrane deformation and working backwards. Using the linearized prediction of the profile then would lead to incorrect conclusions, for instance, to an underestimation of the degree of wrapping.

The energy corresponding to the optimized membrane shape is obtained by inserting the profile (19) back into the functional (16). The necessary integration can be performed analytically, yielding [25]

$$
\widetilde{E}_{\text {free }}=\frac{a}{\lambda}\left(\frac{k^{3}}{1-k^{2}}\right) \frac{K_{0}(k a / \lambda)}{K_{1}(k a / \lambda)} .
$$

\section{Exact asymptotic results}

The small gradient expansion becomes asymptotically exact in the limit of vanishing penetration, $z \rightarrow 0$. It is then also permissible to replace the expression (20) by its small $z$ expansion

$$
\widetilde{E}_{\text {free }}=-2 \widetilde{\sigma} z^{2}\left(2 \gamma+\ln \frac{\widetilde{\sigma} z}{2}\right)+O\left(z^{3}\right)
$$

where $\gamma=0.5772 \ldots$ is the Euler-Mascheroni constant [49]. Up to a (important) logarithmic correction this has the form of a tension energy. In combination with Eq. (4) one obtains the small gradient energy, up to quadratic order in the penetration $z$, and one can discuss the phase behavior.

As already mentioned in Sec. III C, the energy of the free membrane is of higher than linear order for small $z$, hence the transition from free to partially wrapped is dictated by the balance between bending and adhesion alone, giving the phase boundary $\widetilde{w}=4$. The equilibrium penetration follows from $\partial \widetilde{E} / \partial z=0$, which can be rewritten as

$$
W e^{W}=-\frac{\widetilde{w}-4}{8} e^{2 \gamma} \text { with } W:=2 \gamma+\ln \frac{\widetilde{\sigma} z}{2} .
$$

The solution of this transcendental equation is known as the Lambert $\mathcal{W}$ function [50], and in the present situation it is the branch -1 which is needed. One thus obtains

$$
z=\frac{2 e^{-2 \gamma}}{\widetilde{\sigma}} e^{W}=\frac{2 e^{-2 \gamma}}{\widetilde{\sigma} W} W e^{W}=-\frac{\widetilde{w}-4}{4 \widetilde{\sigma} W},
$$

with

$$
W=W(\widetilde{w})=\mathcal{W}_{-1}\left(-\frac{\widetilde{w}-4}{8} e^{2 \gamma}\right)
$$

Equation (23) thus gives the penetration as a function of $\widetilde{w}$ and $\widetilde{\sigma}$. For $x \rightarrow 0^{-}$the function $\mathcal{W}_{-1}(x)$ diverges to $-\infty$ in a logarithmic way [51], therefore the penetration $z(\widetilde{w})$ increases at $\widetilde{w}=4$ for all values of the tension linearly up to a logarithmic correction. Note also the very simple dependence on tension, namely, just inversely proportional.

The expression (23) is of course not valid for all $\widetilde{w}>4$, because the system must also cross the transition toward the enveloped state. At this point one has to make use of a piece of information known from the nonlinear studies. The energy of the free membrane was found to vanish as $z$ approaches 2 , such that only the bound part contributes to the complex energy. Hence, the full envelopment boundary is given by the simultaneous solution of $\partial \widetilde{E} / \partial z=0$ and the additional equation

$$
\widetilde{E}(z)=\widetilde{E}(2)=-2(\widetilde{w}-4)+4 \widetilde{\sigma} .
$$

By eliminating the logarithmic term between those two equations one obtains a quadratic equation for $z$. After inserting its solution into Eq. (23), the final expression can be solved for $\widetilde{\sigma}$, and one arrives at the phase boundary

$$
\widetilde{\sigma}=\frac{\widetilde{w}-4}{4}\left[1+\sqrt{1+\frac{1}{2 W}+\frac{1}{(2 W)^{2}}}\right] .
$$

Remembering the divergence of $W$ as $\widetilde{w} \rightarrow 4^{+}$, Eq. (26) shows that in the limit of weak binding the phase boundary approaches $\widetilde{w}=4+2 \widetilde{\sigma}$, as has been anticipated from the numerical results in Sec. III C (see also Fig. 2). Using the lowest order approximation $\mathcal{W}_{-1}(x) \sim \ln |x|$ at $x \rightarrow 0^{-}$[51] and 
expanding the square root in Eq. (26), one gets the approximate asymptotic phase boundary

$$
\widetilde{\sigma} \simeq \frac{\widetilde{w}-4}{2}\left[1+\frac{1}{8}\left(2 \gamma+\ln \frac{\widetilde{w}-4}{8}\right)^{-1}\right],
$$

which is indicated by the left dotted curve in Fig. 6. The expression (26) is significantly more accurate, but it requires the function $\mathcal{W}_{-1}$ to be evaluated. In any case, since $\mathcal{W}_{-1}(x)$ is only real for $-1 / e \leqslant x<0$, even the full expression (26) exists only up to $\widetilde{w}=4+8 e^{-1-2 \gamma} \approx 4.928$ or, equivalently, $\widetilde{\sigma}=e^{-1-2 \gamma} /(2-\sqrt{3}) \approx 0.433$. At the upper boundary for $\widetilde{\sigma}$ one finds $z=2(2-\sqrt{3}) \approx 0.536$. Larger penetrations than this cannot be described within the quadratic approximation (21) to the small gradient energy (20).

Finally, the value of the penetration $z$ on the discontinuous phase boundary can be obtained by eliminating $\widetilde{w}$ between the two defining equations $\partial \widetilde{E} / \partial z=0$ and Eq. (25). Solving the remaining equation for $\widetilde{\sigma}$ yields

$$
\widetilde{\sigma}=\frac{2}{z} \exp \left\{-\frac{4-z^{2}}{2 z(4-z)}-2 \gamma\right\} \stackrel{z \ll 1}{\simeq} \frac{2}{z} \exp \left\{-\frac{1}{2 z}\right\}
$$

The second approximate relation can also be solved in terms of the Lambert $\mathcal{W}$ function:

$$
\underset{z \stackrel{\sigma}{2} \simeq}{\simeq}-\frac{1}{2 \mathcal{W}_{-1}(-\widetilde{\sigma} / 4)} \stackrel{[51]}{\approx}-\frac{1}{2}\left[\ln \frac{\widetilde{\sigma}}{4}-\ln \left|\ln \frac{\widetilde{\sigma}}{4}\right|\right]^{-1}
$$

In the limit $\widetilde{\sigma} \rightarrow 0$, i.e., when approaching the triple point, the penetration on the discontinuous phase boundary vanishes. Hence, the jump in order parameter approaches 2, i.e., the transition becomes increasingly discontinuous at smaller $\widetilde{\sigma}$. However, Fig. 3 demonstrates that the barrier vanishes in the limit $\widetilde{\sigma} \rightarrow 0$, so from this point of view the transition becomes more continuous. The triple point $(\widetilde{w}=4 ; \widetilde{\sigma}=0)$ is thus quite unusual. Another peculiarity is that along the phase boundary $z$ does not approach 0 in an algebraic way; rather, $z(\widetilde{\sigma})$ has an essential singularity at $\widetilde{\sigma}=0$, as is seen from Eq. (28). All this happens because the small gradient expression for the energy is not a conventional Landau expansion in powers of the order parameter $z$ since the quadratic term has an additional logarithmic factor. This lies at the heart of all logarithmic corrections encountered above (manifest also in the occurrence of the function $\mathcal{W}$ ), and it renders the standard classification schemes for critical points inapplicable here.

The exact asymptotic phase boundary can be obtained, because information about the energy of the fully wrapped state is available. However, for the barrier the situation is different: Even if the equilibrium penetration is very small, the location of the barrier (i.e., the penetration $z_{\text {barrier }}$ at which the energy is largest) occurs at large $z$ (see, e.g., the inset of Fig. 3). In fact, numerical evidence suggests that $\lim _{\tilde{\sigma} \rightarrow 0} z_{\text {barrier }}=1$ from above. It is therefore impossible to obtain the height of the barrier by extending the above small gradient analysis.

\section{SCALING LAWS IN THE HIGH TENSION LIMIT}

As the tension grows, so does the equilibrium penetration on the phase boundary toward the fully enveloped state. The location of this transition can then no longer be obtained within the small gradient framework of the preceding section. Still, the numerical results in Sec. III C point toward a well defined and simple asymptotic behavior in the high tension limit (see, e.g., Fig. 6). Unfortunately, treating the curvature as a small perturbation to the tension is tricky, because this leads to a so-called "boundary layer problem": The solution features a finite variation over a range which vanishes in the perturbative limit [52]. In the present case, the membrane has to bend away from the colloid toward the flat plane [i.e., $\psi(s)$ has to change from $\alpha$ to 0 ] in a region of vanishing arclength. Typically, such problems are dealt with by a subtle matching procedure (an example is provided by the treatment of an ideal neck in Ref. [43]). Somewhat less ambitious, the current section shows how the asymptotic behavior can be quantified by starting with reasonable scaling assumptions about the boundary layer. Still, the resulting formulas will turn out to be remarkably robust.

A useful observation to start with is that for large $\widetilde{\sigma}$ the equilibrium penetration approaches $z=\widetilde{w} / 2 \widetilde{\sigma}$ (see the discussion of the Young-Dupré limit $\kappa \rightarrow 0$ in Sec. III C). Using the contact curvature boundary condition (14), this would predict the asymptotic relation

$$
a \dot{\psi}_{0}=1-\sqrt{\widetilde{w}} \stackrel{\widetilde{\sigma} \gg 1}{\sim}-\sqrt{2 z \widetilde{\sigma}} .
$$

This suspicion is indeed confirmed by a check with the numerical results (data not shown). The proportionality to $\sqrt{\widetilde{\sigma}}$ $=a / \lambda$ is not surprising, since $\lambda$ is the typical length on which the membrane bends. However, the proportionality to $\sqrt{2 z}=2 \sin \alpha / 2$ is not obvious [53].

Equation (30) can be used to infer the asymptotic behavior of several more variables, by virtue of the following scaling argument. Its aim is to estimate the energy of the free part of the membrane, which for large $\widetilde{\sigma}$ is largely stored in a small toroidal rim at contact (this is the boundary layer). This toroid has the axial radius $a \sin \alpha$ and a typical meridinal radius which scales like $1 / \dot{\psi}_{0}$. Up to geometric factors its area is thus proportional to $(a \sin \alpha) / \dot{\psi}_{0}$, and the tension contribution becomes

$$
E_{\text {free }}^{\mathrm{ten}} \sim \sigma \frac{a \sin \alpha}{\dot{\psi}_{0}} \sim \kappa \sqrt{\widetilde{\sigma}} \sqrt{2-z}
$$

The two principal curvatures are $1 / a$ and $\dot{\psi}_{0}$, where the second one clearly dominates in the high tension limit. Hence, the bending energy of this torus scales like

$$
E_{\text {free }}^{\text {bend }} \sim \kappa \frac{a \sin \alpha}{\dot{\psi}_{0}}\left(\dot{\psi}_{0}\right)^{2} \sim \kappa \sqrt{\widetilde{\sigma}} z \sqrt{2-z} \sim \kappa \sqrt{\widetilde{\sigma}} \sqrt{2-z},
$$

where in the last step the prefactor $z$ has been dropped, since for high tension the equilibrium penetration at the transition 


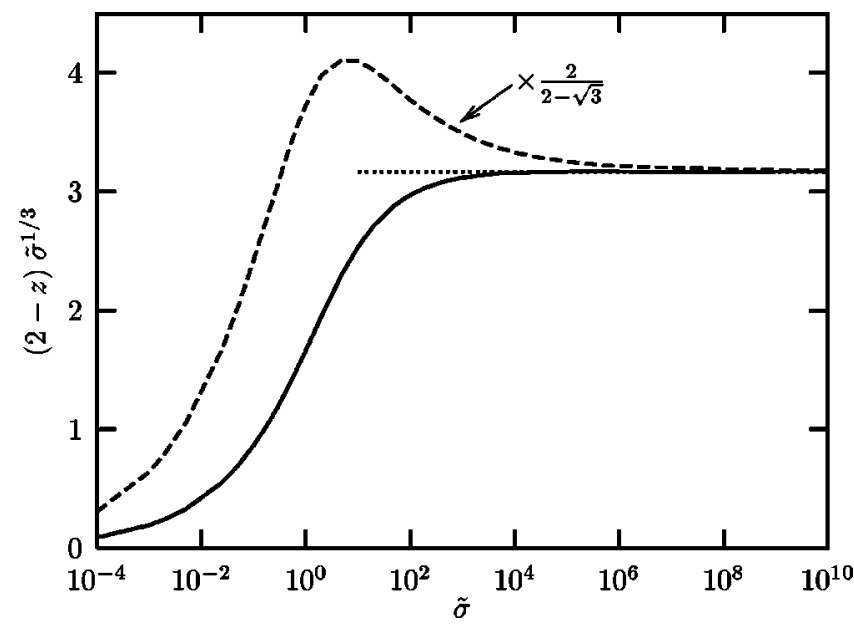

FIG. 8. Scaling plot for the penetration $z$ on the phase boundary (solid) and the location of the barrier, $z_{\text {barrier }}$ (dashed). According to Eqs. (34) and (36) the combination $(2-z) \widetilde{\sigma}^{1 / 3}$ should approach a constant value, which should be the same for both cases if the latter is multiplied by the additional factor $2 /(2-\sqrt{3})$. The dotted line indicates the asymptotic limit $A^{2 / 3} \approx 3.17$.

is close to 2. Equations (31) and (32) show that in the limit of large tension and close to full wrapping the energy of the free membrane can be written as

$$
\widetilde{E}_{\text {free }} \simeq 2 A \sqrt{\widetilde{\sigma}} \sqrt{2-z} \quad(\widetilde{\sigma} \gg 1, z \approx 2)
$$

where the proportionality factor $A$ does not depend on $\widetilde{\sigma}$ or $z$, and the additional factor 2 is included for later convenience.

It is worth pointing out that the $z$ dependence of the scaling form in Eq. (33) can also be understood in the following intuitive way: If one conceives of the strongly curved region at detachment as giving rise to a line energy, $E_{\text {free }}$ ought to be proportional to the length of this line, which is $a \sqrt{z(2-z)}$. For $z$ close to 2 this has the same characteristic variation $\sqrt{2-z}$ as Eq. (33). However, it must be noted that this form holds only in the double limit of large tension and large penetration. Generally, $E_{\text {free }}$ is not well represented by a simple line energy alone [34].

One can now insert the expression (33) into Eq. (4) and discuss the phase behavior. Eliminating $\widetilde{w}$ between the two equations $\widetilde{E}(z)=\widetilde{E}(2)$ and $\partial \widetilde{E}(z) / \partial z=0$ gives the penetration $z$ at the envelopment transition as a function of reduced tension:

$$
\begin{gathered}
\widetilde{\sigma} \gg 1 \\
z 2-A^{2 / 3} \widetilde{\sigma}^{-1 / 3} .
\end{gathered}
$$

The high tension limit of the penetration is thus reached in an algebraic way with an exponent $-1 / 3$; see Fig. 8. Eliminating $z$ instead of $\widetilde{w}$ gives the envelopment boundary:

$$
\frac{\widetilde{w}-4}{\widetilde{\sigma}} \simeq 4-3 A^{2 / 3} \widetilde{\sigma}^{-1 / 3},
$$

showing that its asymptotic value is also reached algebraically with an exponent $-1 / 3$; see Fig. 6.

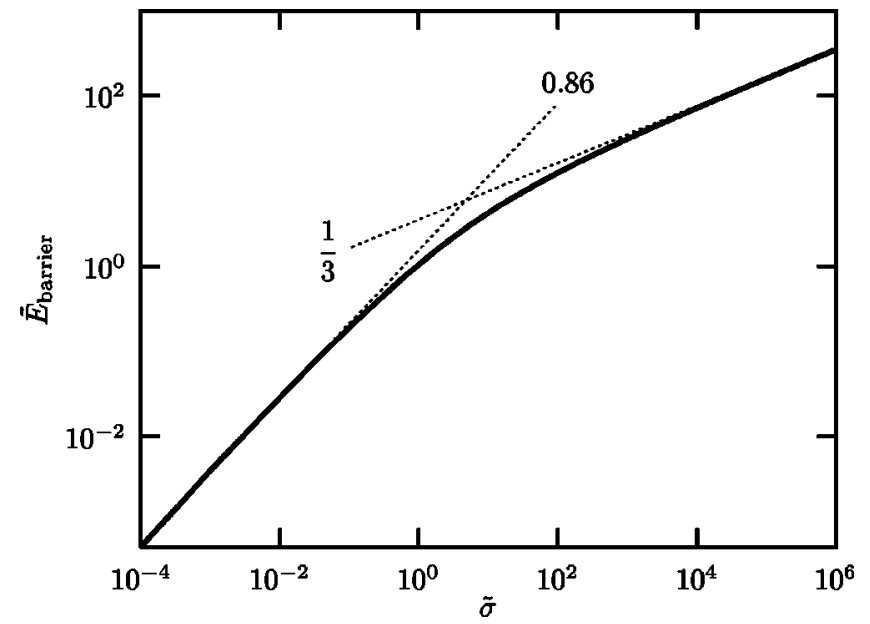

FIG. 9. Barrier height for the transition between partially wrapped and fully enveloped as a function of reduced tension $\widetilde{\sigma}$ on a double logarithmic scale. The two dotted lines indicate the two different regimes: For high tension the barrier height scales with an exponent $1 / 3$, see Eq. (37), while an empirical power law fit to the low tension regime gives the value 0.86 [54].

Information on the barrier can be obtained by further studying the scaling energy. After inserting the phase boundary (35) back into the energy one determines the location of the maximum via $\partial \widetilde{E}(z) / \partial z=0$ :

$$
z_{\text {barrier }} \stackrel{\widetilde{\sigma} \gg 1}{\simeq} 2-\frac{2-\sqrt{3}}{2} A^{2 / 3} \widetilde{\sigma}^{-1 / 3}
$$

The location of the barrier thus reaches the asymptotic value 2 in the same way as the location of the transition, only the prefactor is different by $(2-\sqrt{3}) / 2$. Figure 8 also shows a scaling plot of the location of the barrier, in which this additional factor has been explicitly included. Since both curves in Fig. 8 approach the same limit, the present scaling argument predicts more than the exponent: it correctly predicts the ratio of the prefactors as well.

Finally, the barrier height is determined as the difference between $\widetilde{E}\left(z_{\text {barrier }}\right)$ and $\widetilde{E}(2)$, for which one finds

$$
\widetilde{E}_{\text {barrier }} \stackrel{\widetilde{\sigma} \gg 1}{\simeq} \frac{3}{4}(2 \sqrt{3}-3) A^{4 / 3} \widetilde{\sigma}^{1 / 3} .
$$

This is illustrated in Fig. 9. The two asymptotic power laws meet at the crossover point $\widetilde{\sigma}_{\text {cross }} \approx 4.72$. The numerical value is intriguingly close to the critical tension $\widetilde{\sigma}_{\mathrm{c}}$ mentioned at the end of Sec. III B, but this is probably coincidental.

That the above scaling argument gives the correct relation between the prefactors can also be checked in the following way: Each of the equations (34)-(37) describes a scaling relation for a different variable, but the prefactors all involve $A$. Numerically one can determine $A$ by an asymptotic fit to the high tension values of these four variables, determined from the nonlinear studies of Sec. III. In all cases one finds the same result: $A \approx 5.650$. The scatter among the four results relative to the average value is very small, only about $6 \times 10^{-4}$, and stems most likely from the fitting procedure. 


\section{DISCUSSION OF A BIOLOGICAL EXAMPLE}

In the previous three sections a theoretical description of the adhesion and wrapping behavior between a colloid and a fluid membrane in terms of continuum elasticity theory has been developed, using the full nonlinear shape equations, their small gradient expansion, and scaling arguments. In this final section the results obtained are used to again make contact with a biological application of such wrapping events mentioned in the Introduction, namely, the maturation of animal viruses by budding. It is worth repeating that this event, unlike viral entry, is generally not dependent on metabolic energy [2]. It should thus be reasonably well approximated by the kind of passive wrapping scenario studied in this paper.

While the reduced tension $\widetilde{\sigma}$ used throughout the paper can span many orders of magnitude, it is important to realize that in a biological context the variation is more restricted. Tensions of cellular membranes reported in the literature vary between $0.003 \mathrm{dyn} / \mathrm{cm}$ and $1 \mathrm{dyn} / \mathrm{cm}$ [26]. Larger values soon result in a structural failure of the bilayer. On the other hand, typical bending constants of lipid bilayers are in the range of a few tens up to about a hundred $k_{\mathrm{B}} T[33,47]$. From these numbers one finds a characteristic membrane length $\lambda$ varying roughly between ten and at most a few hundred nanometers. Interestingly, this roughly coincides with the range of particle sizes for which the scenario treated in this paper is biologically meaningful-for quite different reasons: Particles much smaller than $10 \mathrm{~nm}$ are more likely to be transported across a biomembrane by means of channels, while wrapping of particles of a few hundred nanometers or bigger can no longer be described without considering the concomitant significant rearrangements of the actin cortex underlying the lipid bilayer. Moreover, on these larger length scales the shear modulus of the actin network will also play a role, which the current theory for a fluid membrane does not take into account. Hence, if wrapping events of the kind discussed in this paper take place on cellular membranes, they are bound to occur in the regime in which the reduced tension $\widetilde{\sigma}=(a / \lambda)^{2}$ is of order 1 .

A prominent class of colloidal particles exactly within the right range, for which such wrapping events occur and have been studied in great detail, are the nucleoprotein capsids of many animal viruses-belonging, for instance, to the families of Togaviridae, Coronaviridae, Retroviridae, Rhabdoviridae, Ortho- and Paramyxoviridae, and Hepadnaviridae [2]. During their final maturation step the viral capsids are enveloped by a cellular membrane (e.g., the plasma membrane or the endoplasmic reticulum) in an event which is believed to be independent of active cell processes and by which they ultimately leave their host. In the simplest case adhesion is due to a direct interaction between the capsid and the membrane (for instance, in the case of type-D retroviruses [2]). However, more common is an adhesion mediated by viral transmembrane proteins (usually called spikes) which can attach at specific binding sites on the capsid [4-6], and for which Semliki Forest virus (capsid radius $a \approx 30 \mathrm{~nm}, 80$ spikes) is the classical example. Assuming a typical membrane bending stiffness of $\kappa \approx 20 k_{\mathrm{B}} T$, the wrapping bound- ary $\mathrm{W}$ at $\widetilde{w}=4$ corresponds to a binding (free) energy per spike of about $6 k_{\mathrm{B}} T$, which is physically reasonable.

The above estimates indicate that viral budding events can be expected to take place in the low tension regime of the phase diagram, close to the two phase boundaries. This pattern is found time and again in biology: Systems often seem to have evolved to lie close to phase boundaries, because this permits large "effects" to be triggered by comparatively small parameter changes. Recall, however, that the envelopment transition has been found to be discontinuous and associated with a substantial energy barrier, which nature somehow has to overcome. A conceivable solution of this problem would be provided by a coupling between curvature and compositional degrees of freedom [55-58] with the result of enhancing the concentration of lipid species in the highly curved rim which actually prefer a high curvature. This would lower the rim energy and thereby the wrapping barrier. It is also compatible with the finding that the composition of viral membranes frequently deviates from the average cellular lipid composition and resembles that of socalled "raft" domains (for instance, by being rich in cholesterol) $[6,59]$.

It is crucial to realize that it is biologically feasible to actually move in the phase diagram of Fig. 2. For instance, cells actively control and adjust their membrane tension for the purpose of surface area regulation [26]. Even more dramatic changes in tension can occur when one switches between adhering membranes. If viral capsids get spontaneously wrapped, they evidently must be in a region of the phase diagram in which the wrapped state is stable (and, moreover, in which it is not rendered inaccessible by a large barrier). But the virus cannot stay wrapped forever. As it infects a new host cell, it typically becomes internalized via receptor mediated endocytosis and ends up in an endosome, which it again has to leave in order to avoid ultimately being digested by cellular toxins. Many viruses leave the endosome by fusing their outer envelope with the endosomal bilayer, but if the capsid were too strongly attached to the membrane, it could not be freed this way. The biochemical changes within the endosome which lead to the fusion event (in particular, a lowering of $p \mathrm{H}$ ) are usually assumed to also diminish the strength of adhesion. However, within the theoretical framework established in this paper it is tempting to speculate about an alternative mechanism: If the bilayer tension of the endosome is larger than the tension of the membrane at which the capsid became enveloped, unwrapping can be efficiently promoted by vertically crossing the phase boundary E from enveloped to partially wrapped, as can be seen in Fig. 2. Moreover, the horizontal adhesion axis of the phase diagram can not only be changed by chemically modifying the spikes, but also by controlling their density in the membrane [60]. This may not only be relevant in the initial wrapping event, in which an increasing density of spikes in the membrane can push the system over the envelopment boundary, but also in the unwrapping process, when after fusion the spikes can readily diffuse into the essentially spike-free endosomal membrane and thus reduce the binding free energy.

The above example illustrates how the physical principles discussed in this paper can be directly relevant in a biologi- 
cal context. Unfortunately it is often hard to disentangle them from other biological processes or secondary effects of the experimental setup. Hence, a quantitative test of the present work appears more practicable in well controlled model systems, e.g., similar to the ones studied in Refs. $[12,13]$. Nevertheless, the physical results presented here can provide valuable insight into biological problems which may complement other approaches. As an example one might think of a way to measure cellular tension which is an alternative to the current method of pulling a tether [8,23]. The above analysis has shown how the degree of wrapping of a colloid depends on the applied tension-in the regime accessible by the small gradient expansion it is simply inversely proportional, see Eq. (23). One can thus use suitably coated colloids as tension probes. Unlike the tether approach this method is in principle also applicable to intracellular membranes, even though a noninvasive determination of the degree of wrapping will be very difficult for small beads. The theory developed in this work should then be useful for analyzing the results of such measurements.

\section{ACKNOWLEDGMENTS}

It is my pleasure to thank T. Bickel, W. M. Gelbart, S. Tzlil, and A. Ben-Shaul for many stimulating discussions on the subject. Figures 1, 2, 3, and 7 were reproduced from Ref. [25] with kind permission of the publisher. Financial support by the German Science Foundation under Grants Nos. De775/1-1 and De775/1-2 is also gratefully acknowledged.

\section{APPENDIX: CONTACT CURVATURE}

In Sec. III B it was claimed that the boundary condition for the contact curvature, Eq. (14), cannot be enforced if the point of detachment is fixed, but that it holds if additionally the energy is stationary, i.e., $\partial E / \partial z=0$. This statement will be justified below by deriving the boundary condition from the stationarity equation.

The basic idea is that the equilibrium point of detachment is established as a local force balance; or in other words, a variation of the contact point will raise the total complex energy. This variation must leave the contact point on the substrate surface, and Ref. [18] states that this yields the contact curvature in the form of a transversality condition [39]. However, by virtue of locality, for a small variation it suffices to account for the local substrate angle and curvature, which makes it possible to enforce the contact constraint explicitly. Assume, therefore, that at the equilibrium point of detachment the substrate has a slope $\psi_{\mathrm{s}}$, a distance
$r_{\mathrm{S}}$ from the axis, and a meridinal curvature $c_{\mathrm{S}}$ [the azimuthal curvature is then $c_{\mathrm{a}}=\left(\sin \psi_{\mathrm{s}}\right) / r_{\mathrm{s}}$ ]. A variation $\delta s$ of the arclength then entails the following changes in angle, distance, and height of the profile:

$$
\delta \psi=c_{\mathrm{s}} \delta s, \quad \delta r=\cos \psi_{\mathrm{s}} \delta s, \quad \delta h=\sin \psi_{\mathrm{s}} \delta s .
$$

The profile variation implies changes in the energy which stem from the bound and the free part of the membrane. Let us vary the contact point a little bit such that a piece of area $\delta A=2 \pi r_{\mathrm{s}} \delta s$ gets additionally wrapped. This piece will change adhesion, bending, and tension energies, which are easy to calculate since the substrate shape is known. For contributions from the free membrane one needs to look at the boundary terms $[36,39,61]$ which occur upon variation of the functional (5):

$$
\left.\delta \widetilde{E}\right|_{\text {boundary }}=\left[\frac{\partial L}{\partial \dot{\psi}} \delta \psi+\frac{\partial L}{\partial \dot{r}} \delta r+\frac{\partial L}{\partial \dot{h}} \delta h-H \delta s\right]_{\text {boundary }} .
$$

Since a piece of free membrane is removed during this variation, the boundary term (A2) evaluated at $s=0$ contributes with a minus sign. The total change in energy is therefore given by

$$
\begin{aligned}
\delta E= & \left(-w+\frac{1}{2} \kappa\left(c_{\mathrm{s}}+c_{\mathrm{a}}\right)^{2}+\sigma\left(1-\cos \psi_{\mathrm{s}}\right)\right) \delta A \\
& -\pi \kappa\left[2 r_{\mathrm{s}}\left(\dot{\psi}_{0}+c_{\mathrm{a}}\right) \delta \psi+p_{r} \delta r+p_{h} \delta h-H \delta s\right] .
\end{aligned}
$$

Setting $\delta E=0$, inserting the Hamiltonian from Eq. (8) as well as the substrate constraints from Eq. (A1), almost everything cancels, and one obtains the deceptively simple contact curvature condition $\dot{\psi}_{0}=c_{\mathrm{s}}-\sqrt{2 w / \kappa}$ [18], which for spherical substrates becomes Eq. (14). The sign in front of the square root depends on ones choice of the orientation. It is straightforward to check that the condition remains valid if the membrane additionally has a spontaneous curvature. A generalization for the (much harder) case in which no axial symmetry is present has been given by Rosso and Virga [62] and more recently by Capovilla and Guven [63].

Looking for stationary points in $E(z)$ is equivalent to setting the first variation of the contact point to zero, hence it will reproduce the contact curvature condition. However, the route via $E(z)$ has various advantages. For instance, it can distinguish between minima and maxima, it does not overlook boundary minima, and it yields information about barriers.
[1] H. Lodish, A. Berk, S.L. Zipursky, P. Matsudaira, D. Baltimore, and J. Darnell, Molecular Cell Biology (Freeman\&Company, New York, 2000).

[2] H. Garoff, R. Hewson, and D.-J.E. Opstelten, Microbiol. Mol. Biol. Rev. 62, 1171 (1998).

[3] S.B. Sieczkarski and G.R. Whittaker, J. Gen. Virol. 83, 1535 (2002).
[4] H. Garoff and K. Simons, Proc. Natl. Acad. Sci. U.S.A. 71, 3988 (1974).

[5] K. Simons, H. Garoff, and A. Helenius, Sci. Am. 246 (2), 58 (1982).

[6] Y.E. Lu and M. Kielian, J. Virol. 74, 7708 (2000).

[7] O. Boussif, F. Lezoualc'h, M.A. Zanta, M.D. Mergny, D. Scherman, B. Demeneix, and J.-P. Behr, Proc. Natl. Acad. Sci. 
U.S.A. 92, 7297 (1995).

[8] R.M. Hochmuth, J.-Y. Shao, J. Dai, and M.P. Sheetz, Biophys. J. 70, 358 (1996).

[9] R.M. Henderson and H. Oberleithner, Am. J. Physiol. Renal. Physiol. 278, F689 (2000).

[10] M. Abercrombie, J.E.M. Heaysman, and S.M. Pegrum, Exp. Cell Res. 62, 389 (1970).

[11] A. Caspi, O. Yeger, I. Grosheva, A.D. Bershadsky, and M. Elbaum, Biophys. J. 81, 1990 (2001).

[12] C. Dietrich, M. Angelova, and B. Pouligny, J. Phys. II 7, 1651 (1997).

[13] I. Koltover, J.O. Rädler, and C.R. Safinya, Phys. Rev. Lett. 82, 1991 (1999).

[14] R. Lipowsky and H.-G. Döbereiner, Europhys. Lett. 43, 219 (1998).

[15] A.D. Dinsmore, D.T. Wong, P. Nelson, and A.G. Yodh, Phys. Rev. Lett. 80, 409 (1998).

[16] M. Deserno and W.M. Gelbart, J. Phys. Chem. B 106, 5543 (2002)

[17] L.D. Landau and E.M. Lifshitz, Theory of Elasticity, 3rd ed. (Butterworth-Heinemann, Oxford, 1986), Sec. 12, problem 6.

[18] U. Seifert and R. Lipowsky, Phys. Rev. A 42, 4768 (1990).

[19] R. Lipowsky and U. Seifert, Langmuir 7, 1867 (1991).

[20] U. Seifert, Phys. Rev. Lett. 74, 5060 (1995).

[21] J.O. Rädler, T.J. Feder, H.H. Strey, and E. Sackmann, Phys. Rev. E 51, 4526 (1995).

[22] H. Noguchi and M. Takasu, Biophys. J. 83, 299 (2002).

[23] I. Derényi, F. Jülicher, and J. Prost, Phys. Rev. Lett. 88, 238101 (2002).

[24] A. Boulbitch, Europhys. Lett. 59, 910 (2002).

[25] M. Deserno and T. Bickel, Europhys. Lett. 62, 767 (2003).

[26] C.E. Morris and U. Homann, J. Membr. Biol. 179, 79 (2001).

[27] J.-B. Fournier, A. Ajdari, and L. Peliti, Phys. Rev. Lett. 86, 4970 (2001).

[28] M.A. Peterson, J. Appl. Phys. 57, 1739 (1985).

[29] W. Helfrich, Z. Naturforsch. 28c, 693 (1973).

[30] E. Kreyszig, Differential Geometry (Dover, New York, 1991).

[31] The product of the two principal curvatures is called the "Gaussian curvature." Its integral over a surface $S$ can be rewritten as a line integral of the geodesic curvature over the boundary $\partial S$ of $S$ (Gauss-Bonnet theorem [30]). It is thus invariant under deformations of $S$ which change neither its boundary nor its topology. Under these conditions the Gaussian part in Eq. (1) only results in an additive constant in the energy which can be ignored in what follows.

[32] F. David and S. Leibler, J. Phys. II 1, 959 (1991).

[33] Note that the stiffness of a cell membrane depends on the length scale one is probing: For deformations above a few hundred nanometers not just the lipid bilayer but also the underlying actin cortex must deform, leading to bending constants of several hundred $k_{\mathrm{B}} T$-see, for instance, R. Simson, E. Wallraff, J. Faix, J. Niewöhner, G. Gerisch, and E. Sackmann, Biophys. J. 74, 514 (1998). However, on length scales of only a few tens of nanometers, which do not exceed the mesh size of the cortex, the lipid bilayer can deform independently of the actin network and will be characterized by its own, much softer modulus. Note that, for instance, the spectrin network of human erythrocytes has a mesh size of about $75 \mathrm{~nm}$ [see D.
Boal, Mechanics of the Cell (Cambridge University Press, Cambridge, 2002)].

[34] In Ref. [14] it is suggested that for partially wrapped spheres the deformation of the membrane along the contact line will contribute an additional bending term to the contact line energy which acts as an energy barrier for the encapsulation process and which should scale $\sim \sqrt{z(2-z)}$. However, if the membrane is tensionless, such a term is rigorously absent (due to its catenoid shape). Under nonvanishing tension there is indeed a barrier, but it does not have the proposed form, since the bending energy is generally not localized at the rim.

[35] U. Seifert, K. Berndl, and R. Lipowsky, Phys. Rev. A 44, 1182 (1991).

[36] F. Jülicher and U. Seifert, Phys. Rev. E 49, 4728 (1994).

[37] U. Seifert, Adv. Phys. 46, 13 (1997).

[38] Observe that the stronger requirement $h(s) \rightarrow$ const is not used. In fact, for zero tension the solution of the shape equations is the catenoid, which diverges logarithmically in the limit $s$ $\rightarrow \infty$. However, this is a special case, and for any nonzero tension the profile height $h(s)$ remains bounded and converges toward a constant.

[39] R. Courant and D. Hilbert, Methods of Mathematical Physics (Interscience, New York, 1953), Vol. 1.

[40] In several cases of viral budding the nucleoprotein capsid is not preformed but aggregates simultaneously with the budding process [2]. It then imposes a similar boundary condition for the adhering membrane as a complete capsid, except that the membrane is not prevented from having a contact curvature larger than $1 / a$ (there is no capsid yet to exclude this region of space from being occupied). In this case the states with $\dot{\psi}_{0}$ $>1 / a$ are no longer inaccessible and may become physically relevant.

[41] In this paper "low tension" refers to the regime $\widetilde{\sigma} \lesssim 1$. But just because tension is subdominant to curvature, this by no means implies that it is also small compared to the thermal energy $k_{\mathrm{B}} T$. In fact, at the crossover point $\widetilde{\sigma}=1$, the tension energy scales like $E_{\text {ten }} \sim \sigma a^{2}=\kappa$, and $\kappa$ is usually large compared to $k_{\mathrm{B}} T$.

[42] Recall that the adhesion energy contributes a linear term $-\widetilde{w} z$ to the total energy of the complex, see Eq. (4). Increasing $\widetilde{w}$ hence implies "pulling the energy curve $\widetilde{E}(z)$ down at the right-hand side," thus the enveloped state becomes increasingly favorable.

[43] B. Fourcade, L. Miao, M. Rao, M. Wortis, and R.K.P. Zia, Phys. Rev. E 49, 5276 (1994).

[44] As the neck contracts, the membrane deformation will ultimately occur on length scales small compared to the typical size $\lambda$ introduced in Eq. (2), hence tension ceases to be important. But if only bending counts, the optimal solution is the catenoid, which has zero bending energy. Indeed, in the limit $z \rightarrow 2$ the meridinal contact curvature $\dot{\psi}_{0}$ converges toward $-1 / a$, i.e., the negative of the azimuthal contact curvature.

Of course, once the deformation of the membrane occurs on length scales comparable to its thickness (typically a few nanometers), neither linear elasticity theory underlying Eq. (1) nor the description of the membrane as an idealized surface are appropriate, and the bilayer structure needs to be modeled in more detail.

[45] The negative slope of $\widetilde{E}(z)$ at $z=2$ [see inset of Fig. 3] implies 
that the regions of the bilayer at the upper neck are pushed against each other. This may potentially facilitate a subsequent fusion event.

[46] J.S. Rowlinson and B. Widom, Molecular Theory of Capillarity (Dover, New York, 2002).

[47] U. Seifert and R. Lipowsky, in Structure and Dynamics of Membranes, edited by R. Lipowsky and E. Sackmann, Handbook of Biological Physics, Vol. 1A (Elsevier, New York/ North-Holland, Amsterdam, 1995).

[48] Equation (17) can also be derived by a direct small gradient approximation of the shape equations (9). This approach is less elegant, but it illustrates quite vividly how many "unpleasant" terms drop out in lowest order-and only in lowest order. Hence, extending the small gradient expansion to the next order appears about as hard as treating the full nonlinear problem.

[49] Handbook of Mathematical Functions, 9th ed., edited by M. Abramowitz and I.A. Stegun (Dover, New York, 1970).

[50] R.M. Corless, G.H. Gonnet, D.E.G. Hare, D.J. Jeffrey, and D.E. Knuth, Adv. Comput. Math. 5, 329 (1996).

[51] The (essentially) logarithmic divergence is obvious by looking at the inverse of $\mathcal{W}_{-1}(x)$, namely, the function $x e^{x}$, which approaches 0 (essentially) exponentially from the negative side as $x$ approaches $-\infty$. A more precise statement can be obtained by making use of the functional relation $\mathcal{W}_{-1}(x)=\ln |x|$ $-\ln \left|\mathcal{W}_{-1}(x)\right|$, which can be iterated to get increasingly accurate expressions for $\mathcal{W}_{-1}(x)$. Since $\lim _{x \rightarrow 0^{-}} \mathcal{W}_{-1}(x) / \ln |x|=1$, the lowest order reads $\mathcal{W}_{-1}(x) \approx \ln |x|$, and the next correction is then given by $\mathcal{W}_{-1}(x) \approx \ln |x|-\ln |\ln | x||$.

[52] C.M. Bender and S.A. Orszag, Advanced Mathematical Meth- ods for Scientists and Engineers: Asymptotic Methods and Perturbation Theory (Springer, New York, 1999).

[53] One more bit of support stems from Eq. (13), which suggests that $a \dot{\psi}_{0}$ and $\sqrt{1+2 \widetilde{\sigma z}}$ are of the same order. In fact, for $z$ $=1$ one rigorously has $\left|a \dot{\psi}_{0}\right|=\sqrt{1+2 \widetilde{\sigma}}$, for otherwise $p_{r}(0)$ would be infinite.

[54] In the absence of a theoretical description of the barrier height in the low tension regime (a small gradient expansion is not enough), the exponent 0.86 is of course nothing but a purely empirical description of the numerical results, which are equally compatible with the form $a_{1} \widetilde{\sigma} \times \log \left(a_{2} \widetilde{\sigma}\right)$.

[55] S. Leibler, J. Phys. (Paris) 47, 507 (1986).

[56] T. Kawakatsu, D. Andelman, K. Kawasaki, and T. Taniguchi, J. Phys. II 3, 971 (1993); T. Taniguchi, K. Kawasaki, D. Andelman, and T. Kawakatsu, ibid. 4, 1333 (1994).

[57] U. Seifert, Phys. Rev. Lett. 70, 1335 (1993).

[58] F. Jülicher and R. Lipowsky, Phys. Rev. E 53, 2670 (1996).

[59] N. Chazal and D. Gerlier, Microbiol. Mol. Biol. Rev. 67, 226 (2003).

[60] S. Tzlil, M. Deserno, W.M. Gelbart, and A. Ben-Shaul, Biophys. J. (to be published).

[61] The only subtle point is that the arclength $s$ is varied as well, which, for instance, implies that the Hamiltonian $H$ enters into Eq. (A2), and not the Lagrangian $L$. See, for instance, the discussion in H. Goldstein, Classical Mechanics, 2nd ed. (Addison-Wesley, Reading, MA, 1980), Chap. 8-6.

[62] R. Rosso and E.G. Virga, Proc. R. Soc. London, Ser. A 455, 4145 (1999).

[63] R. Capovilla and J. Guven, Phys. Rev. E 66, 041604 (2002). 